\title{
OPEN Elucidation of the core
}

\section{betalain biosynthesis pathway in Amaranthus tricolor}

\author{
Yu-Cheng Chang ${ }^{1}$, Yi-Ching Chiu ${ }^{1}$, Nai-Wen Tsao ${ }^{2}$, Yuan-Lin Chou ${ }^{1}$, Choon-Meng Tan ${ }^{1}$, \\ Yi-Hsuan Chiang ${ }^{1}$, Pei-Chi Liao ${ }^{1}$, Ya-Chien Lee ${ }^{1}$, Li-Ching Hsieh ${ }^{3}$, Sheng-Yang Wang ${ }^{2}$ \& \\ Jun-YiYang ${ }^{1,4,5 \bowtie}$
}

Amaranthus tricolor $\mathrm{L} .$, a vegetable Amaranthus species, is an economically important crop containing large amounts of betalains. Betalains are natural antioxidants and can be classified into betacyanins and betaxanthins, with red and yellow colors, respectively. A. tricolor cultivars with varying betalain contents, leading to striking red to green coloration, have been commercially produced. However, the molecular differences underlying betalain biosynthesis in various cultivars of $A$. tricolor remain largely unknown. In this study, A. tricolor cultivars with different colors were chosen for comparative transcriptome analysis. The elevated expression of AmCYP76AD1 in a red-leaf cultivar of $A$. tricolor was proposed to play a key role in producing red betalain pigments. The functions of AmCYP76AD1, $A m D O D A \alpha 1, A m D O D A \alpha 2$, and AmCDOPA5GT were also characterized through the heterologous engineering of betalain pigments in Nicotiana benthamiana. Moreover, high and low L-DOPA 4,5-dioxygenase activities of AmDODA 1 and AmDODA 2, respectively, were confirmed through in vitro enzymatic assays. Thus, comparative transcriptome analysis combined with functional and enzymatic studies allowed the construction of a core betalain biosynthesis pathway of $A$. tricolor. These results not only provide novel insights into betalain biosynthesis and evolution in $A$. tricolor but also provide a basal framework for examining genes related to betalain biosynthesis among different species of Amaranthaceae.

Betalains are classified into betacyanins and betaxanthins, which provide red-violet and yellow coloration, respectively ${ }^{1}$. Similar to anthocyanins, betalains exhibit antioxidant activity in the form of free-radical scavenging and accumulate in response to different stresses, such as UV-B radiation, high-intensity light, salinity, heat, and drought ${ }^{2-5}$. In addition to their potential roles in protecting plants against abiotic stresses, betalains play a role in defense against pathogenic fungi ${ }^{6}$. Moreover, as water-soluble natural pigments, betalains are widely used as food additives because of their health-promoting properties and color stability over a wide range of $\mathrm{pH}$ values ${ }^{7,8}$.

Betalains occur only in Caryophyllales and have never been detected in anthocyanin-producing plants ${ }^{9,10}$. Although the molecular basis for the mutual exclusion of betalains and anthocyanins is still unclear, breakthroughs in the identification of genes involved in betalain biosynthesis have shed light on the evolution of betalain pigmentation in Caryophyllales ${ }^{9-12}$. Unlike anthocyanins, which are derived from L-phenylalanine, betalains are synthesized from L-tyrosine ${ }^{13,14}$. Initially, L-tyrosine is hydroxylated to produce L-DOPA by tyrosinases encoded by CYP76AD1 and its orthologs ${ }^{15,16}$. L-DOPA can be converted into betalamic acid by L-DOPA 4,5-dioxygenase encoded by DODA, and betalamic acid can then spontaneously condense with amino acids to form betaxanthins ${ }^{1,12,17}$. Alternatively, L-DOPA can be converted into $c y c l o$-DOPA through the oxidase activity of CYP76AD1, and cyclo-DOPA can then spontaneously condense with betalamic acid to form betanidin ${ }^{9,18}$. Betanidin is further glycosylated by betanidin 5-O-glucosyl-transferase encoded by B5GT to form betanin, the most common betacyanin. Glycosylation can also occur on cyclo-DOPA, catalyzed by cyclo-DOPA 5-O-glucosyltransferase encoded by $c D O P A 5 G T$, to produce $c y c l o$-DOPA-glucoside, which then spontaneously condenses with betalamic acid to form betanin ${ }^{10,19}$.

\footnotetext{
${ }^{1}$ Institute of Biochemistry, National Chung Hsing University, 145 Xingda Road, Taichung 40227, Taiwan. ${ }^{2}$ Department of Forestry, National Chung Hsing University, Taichung 402, Taiwan. ${ }^{3}$ Institute of Genomics and Bioinformatics, National Chung Hsing University, Taichung 402, Taiwan. ${ }^{4}$ Institute of Biotechnology, National Chung Hsing University, Taichung 402, Taiwan. ${ }^{5}$ Advanced Plant Biotechnology Center, National Chung Hsing University, Taichung 402, Taiwan. ${ }^{\circledR}$ email: jyang@nchu.edu.tw
} 
a
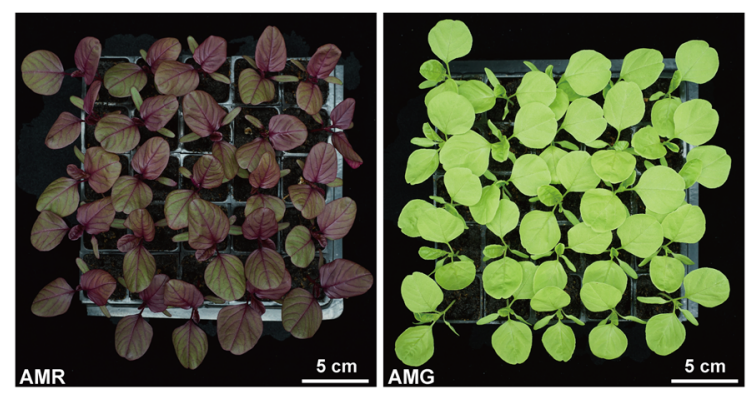

b
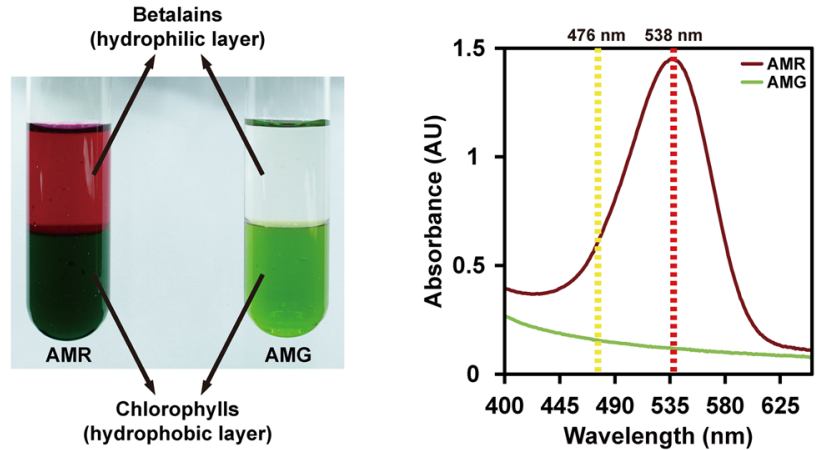

C

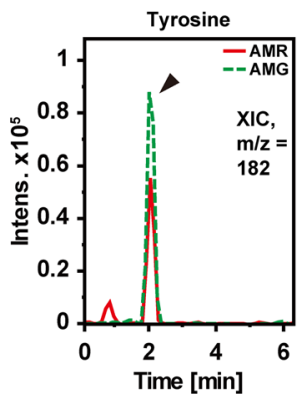

d
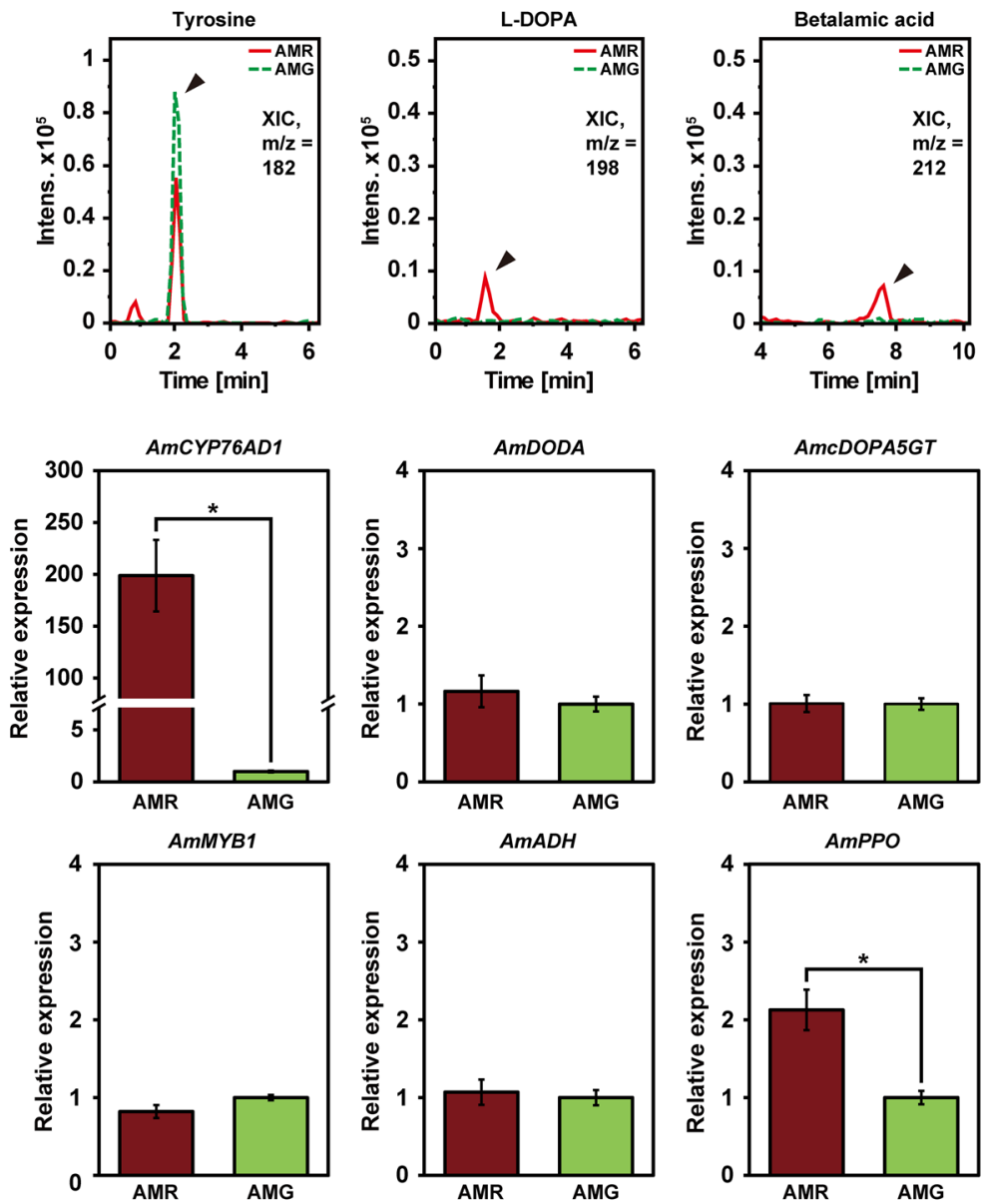
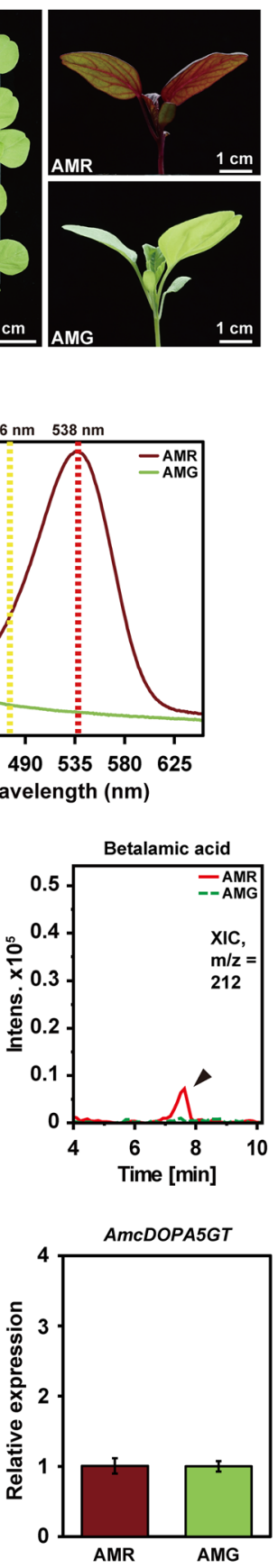

e
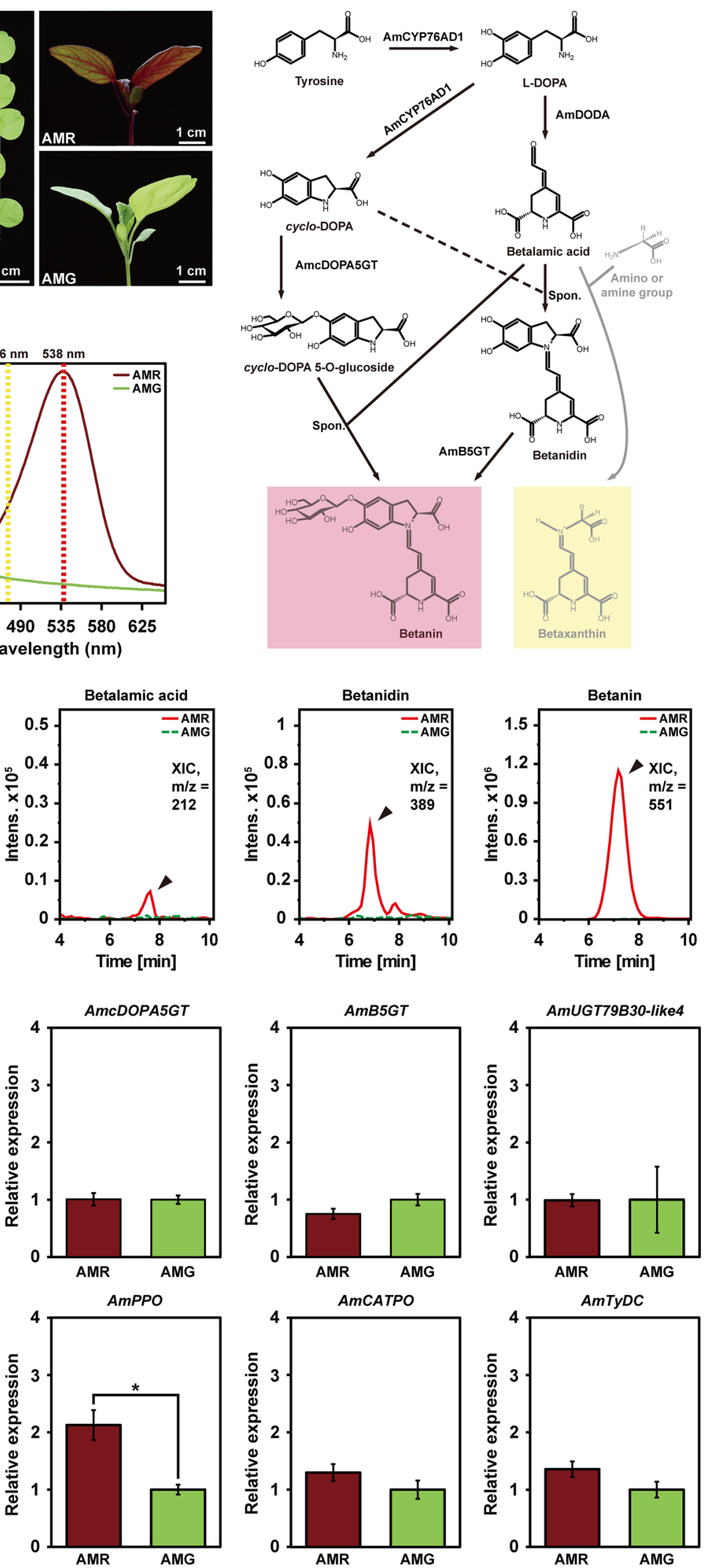
\Figure 1. Identification of $A m C Y P 76 A D 1$ as a key element required for betalain pigment production in Amaranthus tricolor. (a) The leaf-color phenotypes of the red-leaf cultivar (AMR) and green-leaf cultivar (AMG) of three-week-old A. tricolor. (b) Extraction of chlorophyll pigments (hydrophobic layer) and betalain pigments (hydrophilic layer) from three-week-old leaves of AMR and AMG (left panel). Absorbance spectra of the extracted betalain pigments from AMR and AMG (right panel). The absorbance at $538 \mathrm{~nm}$ for betacyanins is indicated with a red dashed line, and the absorbance at $476 \mathrm{~nm}$ for betaxanthins is indicated with a yellow dashed line. (c) Liquid chromatography-tandem mass spectrometry (LC-MS/MS) analysis of three-weekold leaves of AMR and AMG. Shown are extracted ion chromatograms (XICs) of masses corresponding to tyrosine $(\mathrm{m} / \mathrm{z}=182), L$-DOPA $(\mathrm{m} / \mathrm{z}=198)$, betalamic acid $(\mathrm{m} / \mathrm{z}=212)$, betanidin $(\mathrm{m} / \mathrm{z}=389)$, and betanin $(\mathrm{m} / \mathrm{z}=551)$. Time, retention time ( $\mathrm{min})$. (d) Expression levels of genes related to the betalain biosynthesis pathway in AMR and AMG analyzed by qRT-PCR. Statistically significant differences were determined using Student's $t$-test $\left({ }^{\star} P<0.01\right.$ for AMR vs. AMG). (e) Putative core betalain biosynthesis pathway in A. tricolor. Am, Amaranthus tricolor; CYP76AD1, cytochrome P450 76AD1; DODA, DOPA-4,5-dioxygenase; cDOPA5GT, cyclo-DOPA 5-O-glucosyltransferase; B5GT, betanidin-5-O-glucosyltransferase; UGT79B30-like 4, UDP-glucose glucosyltransferase 79B30-like 4; $A D H$, arogenate dehydrogenase; $P P O$, polyphenol oxidase; CATPO, catalasephenol oxidase; TyDC, tyrosine decarboxylase.

Phylogenetic analyses revealed that the CYP76AD and DODA genes, encoding key enzymes in the core biosynthetic pathway of betalains, are highly duplicated in Caryophyllales ${ }^{10,20}$. The CYP76AD gene lineage has undergone at least three duplication events, giving rise to three clades: CYP76Ad $\alpha, C Y P 76 \mathrm{Ad} \beta$ and CYP76AD $\gamma^{9}$. The CYP76Ad $\alpha$ clade includes the CYP76AD1 and CYP76AD3 genes, whose products possess both the tyrosine hydroxylase and L-DOPA 4,5-dioxygenase activities required for L-DOPA and cyclo-DOPA formation, respectively ${ }^{16}$. The CYP76AD $\beta$ clade includes the CYP76AD5, CYP76AD6, and CYP76AD15 genes, which possess only the tyrosine hydroxylase activity required for L-DOPA formation ${ }^{16}$. However, the functions of the genes in the CYP76AD $\gamma$ clade in betalain biosynthesis have not been determined. Duplication events in the DODA gene lineage also gave rise to two major clades: DODA $\alpha$ and DODA $\beta$. The function of DODA $\beta$ is unknown, but the evolution of L-DOPA 4,5-dioxygenase activity in betalain-producing plants was proposed to be led by DODA $\alpha^{9}$. Nevertheless, only one paralogous gene from the DODAa clade shows high L-DOPA 4,5-dioxygenase activity in each species, and others exhibit barely detectable activity ${ }^{12,21}$.

An increased understanding of the betalain biosynthesis pathway has facilitated the metabolic engineering of betalains, providing new sources for basic research studies and commercial applications ${ }^{22,23}$. For example, the fluorescent betaxanthins produced by the expression of $M j D O D A \alpha 1$ in yeast were used as chemical biosensors to reveal the tyrosine hydroxylase activity of BvCYP76AD $1^{15}$. The production of semisynthetic betaxanthins by spontaneous condensation between fluorescent betalamic acid and the amino groups of proteins provides an alternative method for labeling proteins for multiple biological applications ${ }^{24}$. Fine-turning the content ratio of betacyanins and betaxanthins via the differential expression of BvCYP76AD1 and BvCYP76AD6 in nonCaryophyllales makes it possible to create a range of color patterns in the background of anthocyanin-producing plants $^{6}$. The heterologous production of betalains via the coexpression of $B v C Y P 76 A D 1, B v D O D A \alpha 1$, and $M j c-$ $D O P A 5 G T$ in tobacco enhances the resistance of transgenic plants to Botrytis cinereal infection ${ }^{6}$. In addition, betanin rice generated through the coexpression of meloS, BvCYP76AD1, and BvDODA $\alpha 1$ in rice endosperm shows higher antioxidant activity and can be provided as a functional food ${ }^{25}$.

Amaranth species are economically important crops containing large amounts of betalains. They can be classified into three categories: vegetable Amaranthus, grain Amaranthus, and weed Amaranthus species ${ }^{26}$. Amaranthus tricolor L., a vegetable Amaranthus species, is widely distributed in warm and tropical regions and is cultivated as a leafy vegetable. As rich in natural antioxidants, A. tricolor is able to tolerate abiotic stresses and has been used as a traditional Chinese medicinal herb for the treatment of eruptive fever, pain, sore throat, dysentery, anemia, bronchitis, colic, $\operatorname{etc}^{27}$. A. tricolor cultivars with various contents of betacyanins and betaxanthins, resulting in striking colors ranging from red to green, have been commercially produced ${ }^{27,28}$. However, the molecular basis underlying betalain biosynthesis in A. tricolor remains largely unknown. Recently, virus-induced gene silencing was applied to elucidate the function of $C Y P 76 A D 1$ in producing betalain pigments in $A$. tricolor ${ }^{29}$. In addition, a comparative analysis of a transcriptome database constructed from different leaf samples of $A$. tricolor cv. Dahong was performed to construct a putative metabolic pathway of betalains in A. tricolor ${ }^{30}$. Candidate genes encoding enzymes catalyzing the formation of L-DOPA, cyclo-DOPA, betalamic acid, cyclo-DOPA-glucoside, and betanin were obtained from a transcriptome database and showed higher expression levels in red areas of A. tricolor leaves than in in green areas ${ }^{30}$. However, functional characterization and enzyme activity analyses are still needed to elucidate the roles of these candidate genes in the betalain biosynthesis pathway of A. tricolor.

In this study, A. tricolor cultivars with different colors were chosen for comparative transcriptome analysis. The key gene showing elevated expression in a red-leaf cultivar of $A$. tricolor was identified, and the results indicated that the dual activities of tyrosine hydroxylase and L-DOPA oxidase are important for producing red betalain pigments in A. tricolor. The core betalain biosynthesis pathway of A. tricolor was further constructed based on the functional characterization of betalain biosynthesis genes through the heterologous engineering of betalain pigments in Nicotiana benthamiana and in vitro enzymatic assays of L-DOPA 4,5-dioxygenase activities. These results provide novel insights into betalain biosynthesis and evolution in A. tricolor.

\section{Results}

AmCYP76AD1 is highly expressed in a red-leaf cultivar of $\boldsymbol{A}$. tricolor. A. tricolor cultivars are important leafy vegetables that display leaf colors ranging from red to green depending on the betalain content ${ }^{28}$. To elucidate the genetic factors that influence betalain pigment contents in red-and green-leaf cultivars of $A$. tri- 
color (hereafter referred to as AMR and AMG, respectively) (Fig. 1a-c, Supplementary Fig. S1a, b), specific primer pairs were designed based on available sequence information from the NCBI database or previously published studies to selectively examine the transcript levels of genes related to the betalain biosynthesis pathway by qRTPCR (Supplementary Table S1). Among them, AmADH, AmCYP76AD1, AmDODA, AmcDOPA5GT, AmB5GT, and AmUGT79B30-like 4 genes encoding for the putative enzymes were thought to be directly involved in the betalain biosynthesis ${ }^{30}$. AmMYB1, encoding an R2R3-type transcription factor, was proposed to participate in the regulation of betalain biosynthesis ${ }^{10,30}$. AmPPO and AmCATPO genes, encoding tyrosinases, were considered for their roles in conversion of L-DOPA to cyclo-DOPA ${ }^{1,30}$. AmTyDC, encoding a tyrosine decarboxylase, was proposed to participate in the degradation of betalain ${ }^{1,30}$. In 3-week-old A. tricolor, AmCYP76AD1 and AmPPO showed higher expression levels in AMR than in AMG (Fig. 1d). Notably, only AmCYP76AD1 exhibited a highly differential expression pattern, showing an 200-fold difference between AMR and AMG. In contrast, AmDODA, AmcDOPA5GT, AmB5GT, AmUGT79B30-like 4, AmMYB1, AmADH, AmCATPO, and AmTyDC did not show a significant differential expression pattern between AMR and AMG (Fig. 1d). The highly differential expression pattern of AmCYP76AD1 between AMR and AMG was also observed in 4-week-old A. tricolor (Supplementary Fig. S1c). Moreover, as a key element in the initiation of the betalain biosynthesis pathway, AmCYP76AD1 displayed higher transcript levels in the upper leaves of AMR, which contained higher content of betalains than those in the lower leaves of AMR (Fig. 2a,b, Supplementary Fig. S2). Further phylogenetic reconstruction and LOGO analysis revealed that AmCYP76AD1 belongs to the CYP76Ada clade (Fig. 2c,d), whose members possess both the tyrosine hydroxylase and L-DOPA oxidase activities required for L-DOPA and cycloDOPA formation, respectively (Fig. 1e). These results suggest that the elevated expression of AmCYP76AD1 is necessary for betalain pigment accumulation, which leads to an obvious red-violet color in the leaves and stems of AMR, but not in those of AMG.

AmDODA exhibits a marginal level of L-DOPA 4,5-dioxygenase activity. Although candidate transcripts related to betalain biosynthesis were identified previously in A. tricolor ${ }^{30,31}$, their functional and enzymatic activities have not yet been characterized. To functionally characterize the enzyme activities of AmCYP76AD1, AmDODA, and AmcDOPA5GT in the core pathway of betalain biosynthesis (Fig. 1e), 35S promoter-driven cDNAs encoding C-terminal YFP- or FLAG (SFP)-tagged AmCYP76AD1, AmDODA, and AmcDOPA5GT were transiently coexpressed in N. benthamiana leaves by agroinfiltration. Upon expression, only a small amount of betalain pigment was produced in $N$. benthamiana leaves, which was barely detectable (Fig. 3a). In contrast, as a positive control, high production of betalain pigments with red-violet color was observed when the Beta vulgaris tyrosinase gene (BvCYP76AD1), the B. vulgaris L-DOPA 4,5-dioxygenase gene $(B v D O D A \alpha 1)$, and the Mirabilis jalapa cyclo-DOPA 5-O-glucosyltransferase gene (MjcDOPA5GT), were coexpressed in $N$. benthamiana leaves (Fig. 3a). To elucidate the A. tricolor genes responsible for the negligible activity of betalain synthesis in transient analysis, a series of coinfiltration assays were carried out by replacing the positive control genes individually with AmCYP76AD1, AmDODA, and AmcDOPA5GT. The replacements of $B v C Y P 76 A D 1$ and MjcDOPA5GT by AmCYP76AD1 and AmcDOPA5GT, respectively, resulted in high amounts of betalain pigment and betanin production in $N$. benthamiana leaves (Fig. 3b,c). However, AmDODA failed to replace the function of $B v D O D A \alpha 1$. The coexpression of $B v C Y P 76 A D 1, A m D O D A$, and MjcDOPA5GT only produced marginal levels of betalain pigments and betanin, which were barely detectable (Fig. 3b,c). Together with the comparable levels of proteins detected by western blotting (Fig. 3d, Supplementary Fig. S3), these results suggest that the L-DOPA 4,5-dioxygenase activity of AmDODA is very low compared to that of BvDODAa1.

Two DODA $\alpha$ homologues are present in A. tricolor. Recently, a phylogenetic study of Caryophyllales suggested that at least two $D O D A \alpha$ genes are present in betalain-pigmented species, including Amaranthus hypochondriacus ${ }^{12}$. To identify the DODAa homologue exhibiting a high level of L-DOPA 4,5-dioxygenase activity in A. tricolor, the RNA sequencing of aerial tissues derived from AMR and AMG plants was performed on the Illumina HiSeq 4000 platform. Two transcript libraries of AMR and AMG were built from the highquality reads through de novo assembly and functional annotation (Supplementary Tables S2, S3). The relative abundance of transcripts between AMR and AMG was illustrated in an MA plot (Fig. 4a). In addition, the relevant genes involved in the synthesis of betalain pigments were identified through in silico analysis and further highlighted in the MA plot (Fig. 4a, Supplementary Table S4). As expected, only AmCYP76AD1 was expressed at a significantly higher level in AMR than in AMG (Fig. 4a). These results suggest that AmCYP76AD1 is the key enzyme responsible for betalain pigment accumulation in AMR and that the loss of AmCYP76AD1 expression in AMG results in the green color phenotype.

Additionally, two DODA $\alpha$ homologues, $A m D O D A \alpha 1$ and $A m D O D A \alpha 2$ (referred to as AmDODA), were recovered through in silico analysis (Supplementary Fig. S4, Table S4). This indicated that gene duplication has occurred at least once in the DODA $\alpha$ lineage of A. tricolor. A reduced phylogenetic tree of DODA $\alpha$ was further generated using AmDODAa1, AmDODAa2, and previously characterized DODAa homologues from B. vulgaris, Carnegiea gigantea, Chenopodium quinoa, Mesembryanthemum crystallinum, M. jalapa, Parakeelya mirabilis, and Stegnosperma halimifolium (Fig. 4b). Two clades, DODAa1 and DODAa2, were obtained, and each of them presented seven previously identified conserved residues that are functionally important for high and marginal activities of L-DOPA 4,5-dioxygenase, respectively (Fig. 4c). Among these sequences, AmDODAa1 belongs to the DODAal clade and contains seven residues (DDYNDEI) associated with high L-DOPA 4,5-dioxygenase activity; AmDODAa2 (AmDODA) belongs to the DODAa2 clade and contains seven residues (YGFKNNT) associated with marginal L-DOPA 4,5-dioxygenase activity. These results suggest that AmDODAa1 may exhibit the high level of L-DOPA 4,5-dioxygenase activity required for betalain pigment production in A. tricolor. 
a
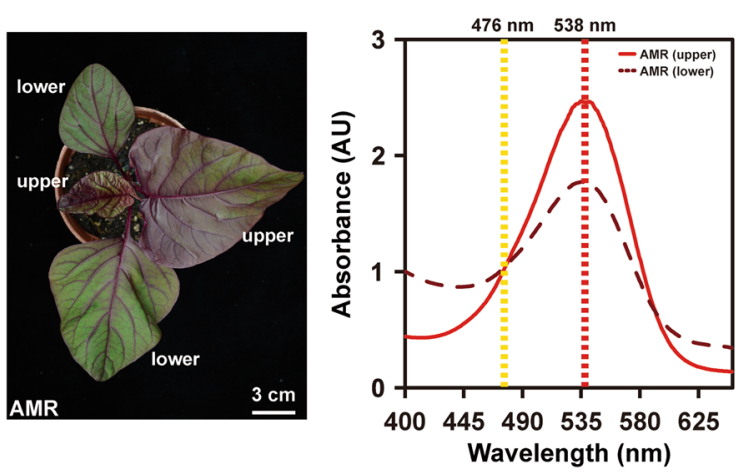

b
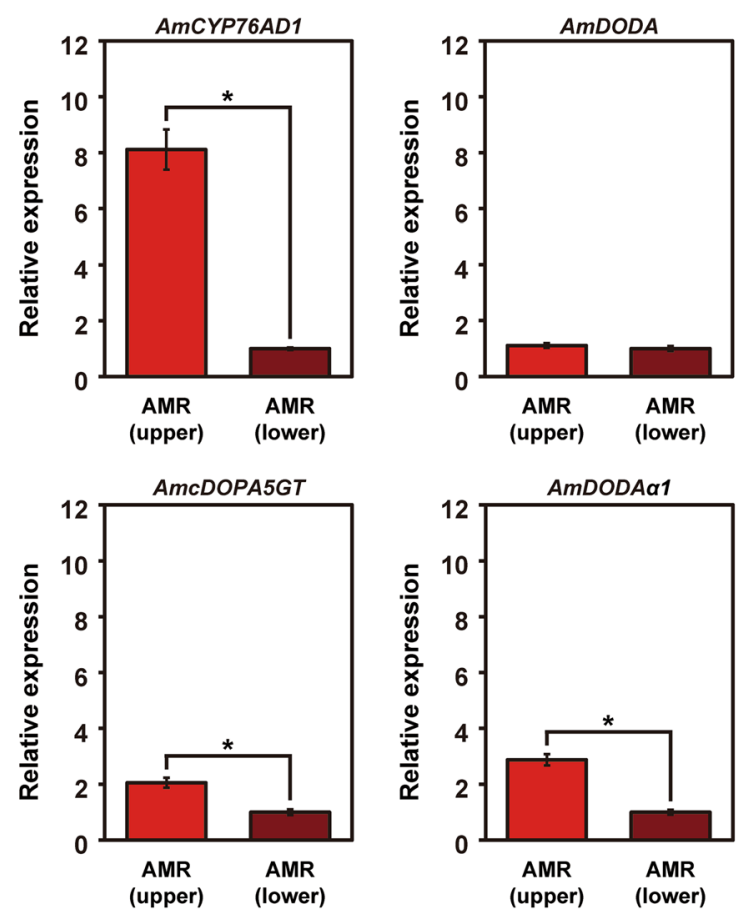

C

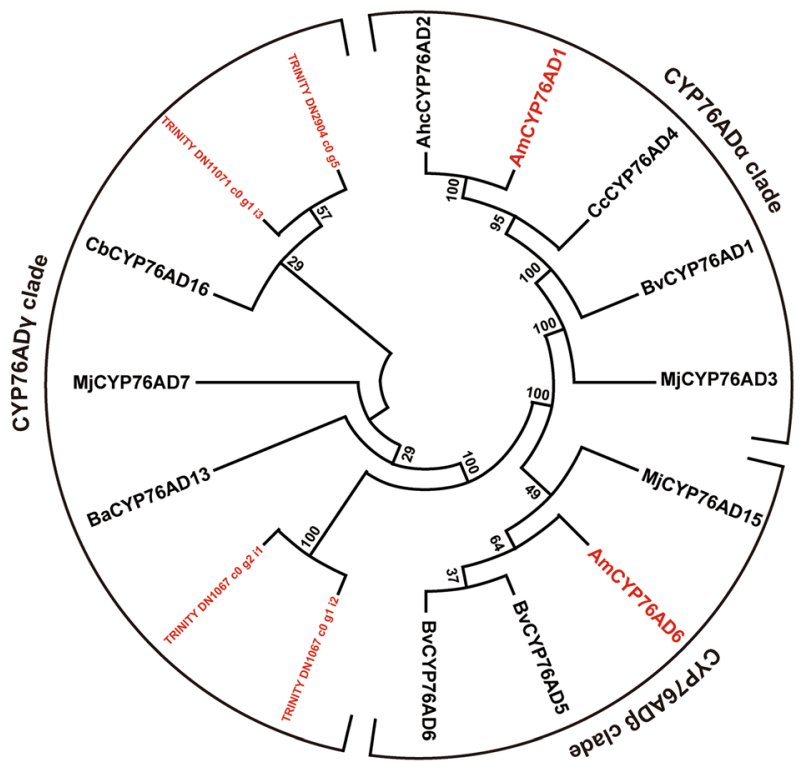

d

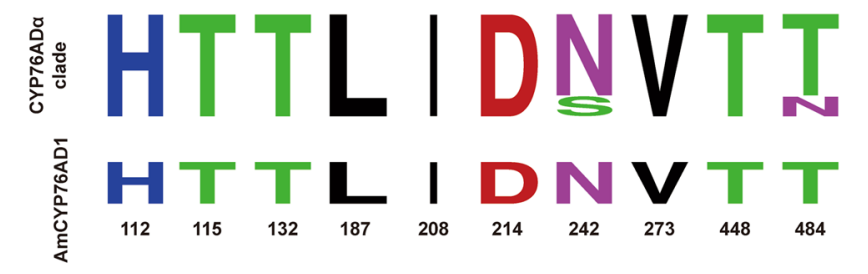

IDSSTMEYTNY

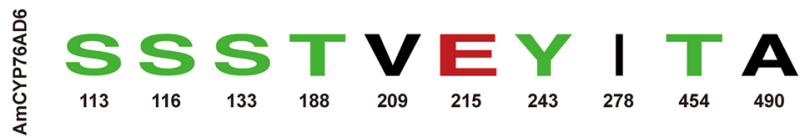

Figure 2. Expression pattern, phylogenetic reconstruction, and LOGO analysis of AmCYP76AD1. (a) The leaf color phenotypes and betalain absorbance spectra of the upper and lower leaves of the red-leaf cultivar (AMR) of four-week-old A. tricolor. (b) Expression levels of core betalain biosynthesis genes in the upper and lower leaves of four-week-old AMR plants analyzed by qRT-PCR. Statistically significant differences were determined using Student's $t$-test $\left({ }^{*} P<0.01\right.$ for the upper leaves of AMR vs. the lower leaves of AMR). (c) Phylogenetic tree of CYP76AD homologues. The species, families, and accession numbers of CYP76AD homologues are available in Supplementary Table S6. (d) Proportional LOGO plots of selected amino acids identified by Brockington et $\mathrm{al}^{9}$ were generated based on the CYP76AD $\alpha$ and CYP76AD $\beta$ homologues listed in (c). Positions are numbered according to the residues of AmCYP76AD1 and AmCYP76AD6.

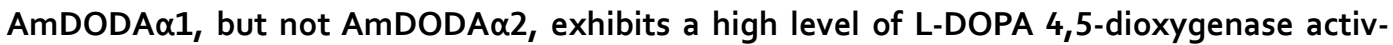
ity. As a key step in betalain biosynthesis, L-DOPA 4,5-dioxygenase can convert L-DOPA into betalamic acid, the basic structural unit of all betalains ${ }^{1,32}$. To functionally characterize the L-DOPA 4,5-dioxygenase activity of AmDODAa1, AmDODA $\alpha 1$ was coexpressed with BvCYP76AD1 and MjcDOPA5GT by agroinfiltration. As a result, high production of betalain pigments and betanin was observed when comparable amounts of proteins were expressed in N. benthamiana leaves (Fig. 3b-d, Supplementary Fig. S3). These results indicate that AmDODAa1, but not AmDODAa2, exhibits a high level of L-DOPA 4,5-dioxygenase activity, similar to that of BvDODAa1.

To verify enzyme activity in vitro, AmDODAa1 and AmDODAa2 were expressed as SUMO-fused recombinant proteins in an Escherichia coli expression system (Fig. 5a). Enzymatic reactions were conducted following the method described by Sasaki et $\mathrm{al}^{32}$, in which crude extracts prepared from $E$. coli were used. After incubation for $5 \mathrm{~min}$ at $30^{\circ} \mathrm{C}$, a bright yellow color derived from betalamic acid was observed in the reaction mixture containing L-DOPA, ascorbic acid, and a crude extract prepared from E. coli harboring AmDODA $\alpha 1$ or $B v D O D A \alpha 1$, but not $A m D O D A \alpha 2$ (Fig. 5b). However, only a very weak yellow color was observed when the reaction mixture contained twofold crude extract prepared from E. coli harboring AmDODA $\alpha 2$ (Fig. 5b). As a control, a reaction mixture containing the crude extract was prepared from $E$. coli harboring only the vector, and no color was 
a

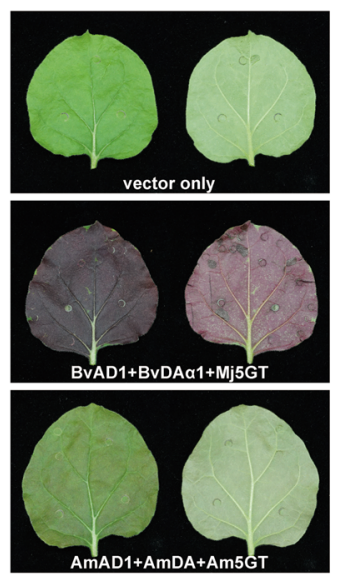

b
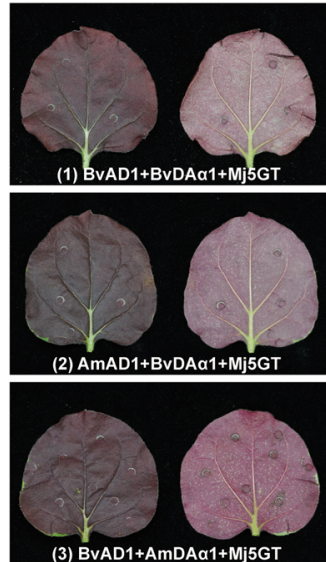
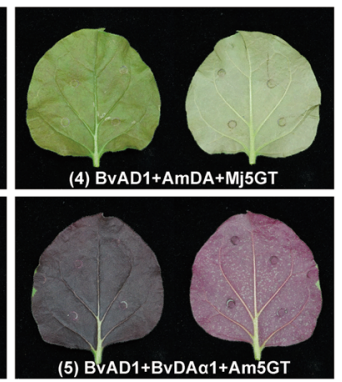

(1) (2) (3) (4) (5)

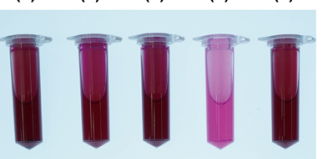

d

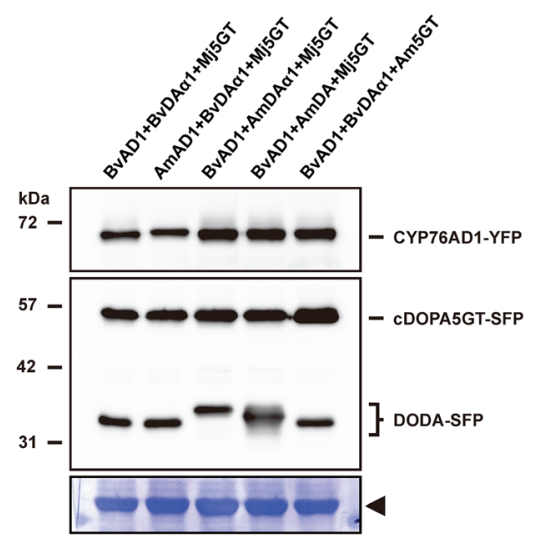

C

BvAD1+BvDAa1+Mj5GT
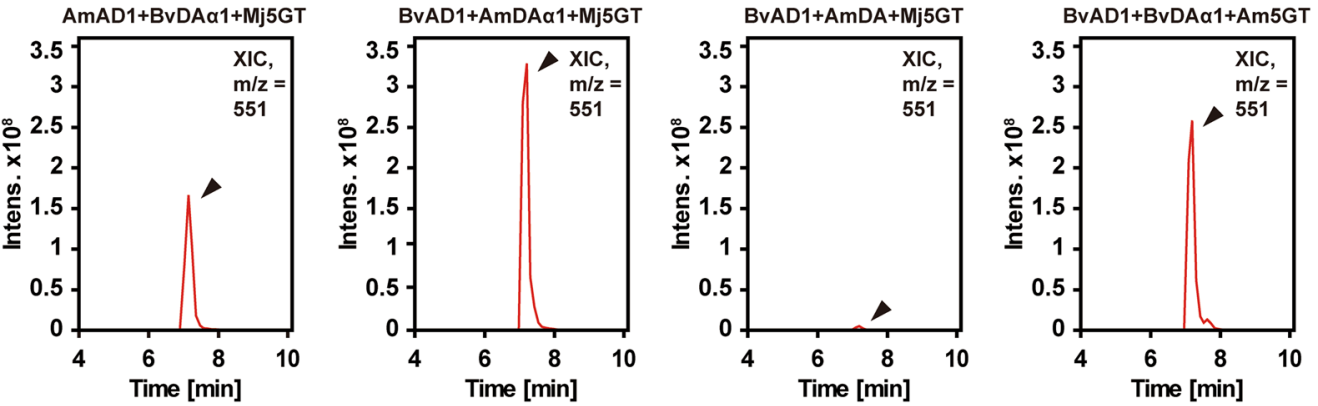

Figure 3. Functional characterization of the enzyme activities of AmCYP76AD1, AmDODA, and AmcDOPA5GT by agroinfiltration for the heterologous engineering of betalain pigments in Nicotiana benthamiana. (a, b) N. benthamiana leaves coinfiltrated with Agrobacterium harboring plasmids for the expression of $B v C Y P 76 A D 1-Y F P(B v A D 1), B v D O D A \alpha 1-S F P(B v D A \alpha 1)$, MjcDOPA5GT-SFP (Mj5GT), $A m C Y P 76 A D 1-Y F P(A m A D 1)$, AmDODA-SFP (AmDA), and AmcDOPA5GT-SFP (Am5GT). Both the adaxial (left) and abaxial (right) sides of leaves are presented in each panel. Bottom right corner indicated the betalain pigments produced in N. benthamiana. (c) Extracted betalain pigments were examined for betanin content by LC-MS/MS analysis. Shown are XICs of masses corresponding to betanin $(\mathrm{m} / \mathrm{z}=551)$. Time, retention time (min). (d) Western blotting assays were conducted with antibodies against the YFP- or FLAG (SFP)-tag to examine the expression levels of YFP-tagged CYP76AD1 (upper panel), SFP-tagged DODA (middle panel), and SFP-tagged cDOPA5GT (middle panel). As a loading control, the large subunit of Rubisco visualized by Coomassie brilliant blue staining is indicated by the arrowhead (lower panel).

observed (Fig. 5b). The reaction products were then subjected to LC-MS/MS analysis and revealed that the clear peak at a retention time of $7.5 \mathrm{~min}$ was betalamic acid (Fig. 5c). These results confirm that AmDODAa2 exhibits marginal levels of L-DOPA 4,5-dioxygenase activity.

Reconstruction of the core betalain biosynthesis pathway of $A$. tricolor in $N$. benthamiana. In this study, we also attempted to use TRV-based virus-induced gene silencing (VIGS) to examine the functional activities of genes involved in betalain biosynthesis in A. tricolor. However, the transient silencing of $A m C Y$ $P 76 A D 1$ in $A$. tricolor was particularly challenging and failed in our hands. In addition, the attempted overexpression of $A m C Y P 76 A D 1$ to complement the betalain pigments in the leaves of AMG was unsuccessful using an agroinfiltration system. These differences might have resulted from the different varieties and low transformation efficiency of $A$. tricolor $^{33}$.

To reconstruct the core betalain biosynthesis pathway of A. tricolor, AmCYP76AD1, AmDODA 1 , and Amc$D O P A 5 G T$ were transiently overexpressed in N. benthamiana leaves by agroinfiltration for the heterologous engineering of betalain pigments. Similar to the vector-only control, the heterologous expression of single $A m C Y$ P76AD1, AmDODA $\alpha 1$, or AmcDOPA5GT was not sufficient to produce any betalain pigment in N. benthamiana (Fig. 6a). However, low production of betalain pigments was observed when AmCYP76AD1 and AmDODA 1 were coexpressed in $N$. benthamiana (Fig. 6a). In contrast, no betalain pigment was observed when $A m C Y$ P76AD1 and AmcDOPA5GT or AmDODA $\alpha 1$ and AmcDOPA5GT were coexpressed in N. benthamiana (Fig. 6a). Only the coexpression of $A m C Y P 76 A D 1, A m D O D A \alpha 1$, and $A m c D O P A 5 G T$ together was sufficient to produce high amounts of betalain pigments in N. benthamiana, which resulted in a strong red-violet color (Fig. 6a). The strong red-violet color was similar to that in the positive control in which $B v C Y P 76 A D 1, B v D O D A \alpha 1$, and MjcDOPA5GT were coexpressed in N. benthamiana (Fig. 6a). As expected, the coexpression of $A m C Y$ $P 76 A D 1, A m D O D A \alpha 2$, and AmcDOPA5GT only produced marginal levels of betalain pigments, which were 
a

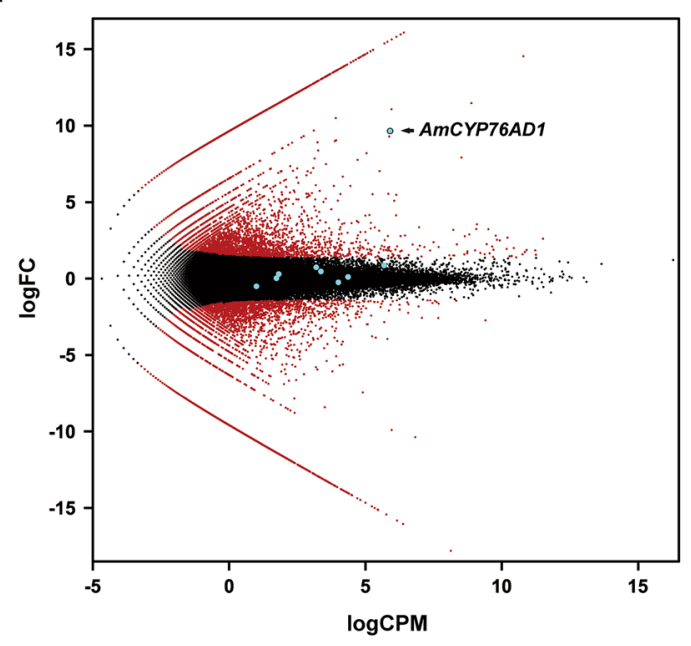

C

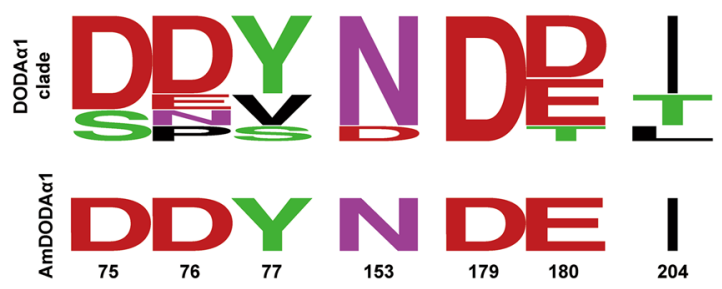

b

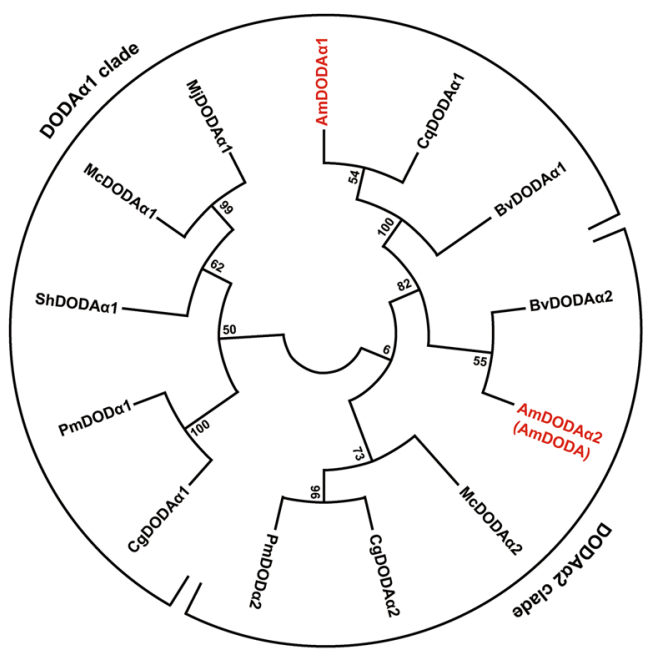

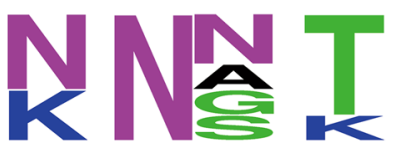

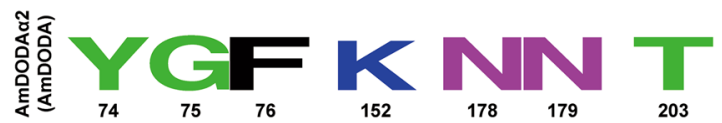

Figure 4. In silico analysis of relevant genes involved in the betalain biosynthesis pathway of $A$. tricolor. (a) The relative abundance of transcripts between AMR and AMG is presented in the MA plot (logCPM vs. $\log F C)$. Each dot presents a gene, and the relevant genes associated with betalain pigment synthesis are highlighted. (b) Phylogenetic tree of functionally characterized DODAa homologues. The species, families, and accession numbers of the DODAa homologues are available in Supplementary Table S6. (c) Proportional LOGO plots of seven functionally important residues identified by Bean et al. $(2018)^{21}$ were generated based on the DODAal and DODAa 2 homologues listed in (b). Positions are numbered according to the residues of AmDODAa1 and AmDODAa2.

barely detectable (Fig. 6a). Consistently, high production of betanin was observed only when AmCYP76AD1, $A m D O D A \alpha 1$, and AmcDOPA5GT were coexpressed in N. benthamiana leaves (Fig. 6b). Together with the comparable amount of proteins detected by western blotting (Fig. 6c, Supplementary Fig. S5), our results suggest that the enzyme activities of AmCYP76AD1, AmDODAa1, and AmDOPA5GT are sufficient to construct the core betalain biosynthesis pathway of $A$. tricolor.

\section{Discussion}

Molecular genetics have shed light on the betalain biosynthesis pathway and its evolutionary significance in Caryophyllales. Based on phylogenetic analysis, CYP76AD homologues can be classified into $\alpha, \beta$, and $\gamma$ clades ${ }^{9}$. To date, only the functions of CYP76ADa and CYP76AD $\beta$ clade homologues, such as CYP76AD1 and CYP76AD6, have been reported ${ }^{10}$. For example, the cosilencing of CYP76AD1 and CYP76AD6 represses the production of betacyanins and betaxanthins in $B$. vulgaris, causing a green leaf phenotype ${ }^{16}$. In this study, a CYP76AD6-like (AmCYP76AD6) gene, belonging to the CYP76AD $\beta$ clade according to phylogenetic construction and LOGO analysis (Fig. 2c,d), was also identified in A. tricolor through transcriptome analysis (Supplementary Fig. S6). However, the expression of AmCYP76AD6 was extremely low and was difficult to detect in AMR and AMG. As a result, it is difficult to functionally connect $A m C Y P 76 A D 6$ with the production of betalains in $A$. tricolor. In addition, although $P P O$, a polyphenol oxidase gene, and $C A T P O$, a catalase-phenol oxidase gene, were previously proposed to be involved in betalain biosynthesis via monophenolase activity ${ }^{34,35}$, their transcripts did not show highly differential expression patterns between AMR and AMG (Fig. 1d, Supplementary Fig. S1c). As a result, we propose that the elevated expression of $A m C Y P 76 A D 1$ is necessary for the occurrence of a red-violet color phenotype in A. tricolor; in contrast, the loss of $A m C Y P 76 A D 1$ expression results in a green color phenotype in $A$. tricolor (Fig. 1a-d). The existence of the AmCYP76AD1 gene in AMG examined by PCR using genomic DNA as a template confirmed the loss of $A m C Y P 76 A D 1$ expression in AMG (Supplementary Fig. S7). Together with the functional characterization of the enzymatic activity of AmCYP76AD1 through the heterologous engineering of betalain pigments in $N$. benthamiana (Figs. 3b, 6a), we conclude that AmCYP76AD1, a CYP76ADa homologue required for the initiation of the betalain biosynthesis pathway, plays a key role in betalain pigment accumulation in A. tricolor. Accordingly, AmCYP76AD1 displayed higher transcript levels in the upper leaves of AMR, which contained higher content of betalains than those in the lower leaves of AMR (Fig. 2a,b, Supplementary Fig. S2).

In recent years, with the elucidation of the central committed steps of the betalain biosynthesis pathway, comparative transcriptome analyses have been intensively applied to identify genes involved in regulating betalain 
a

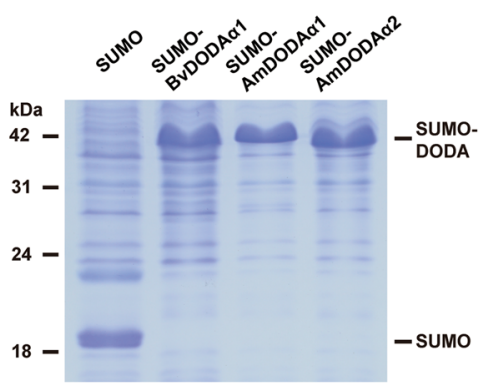

b

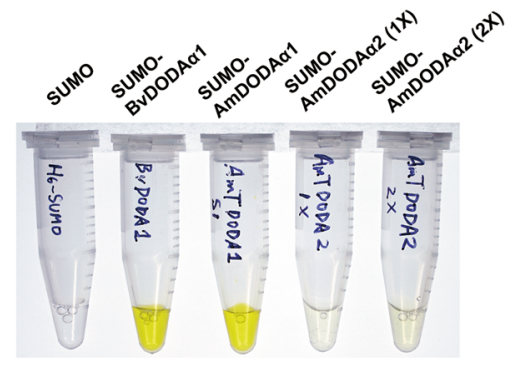

C
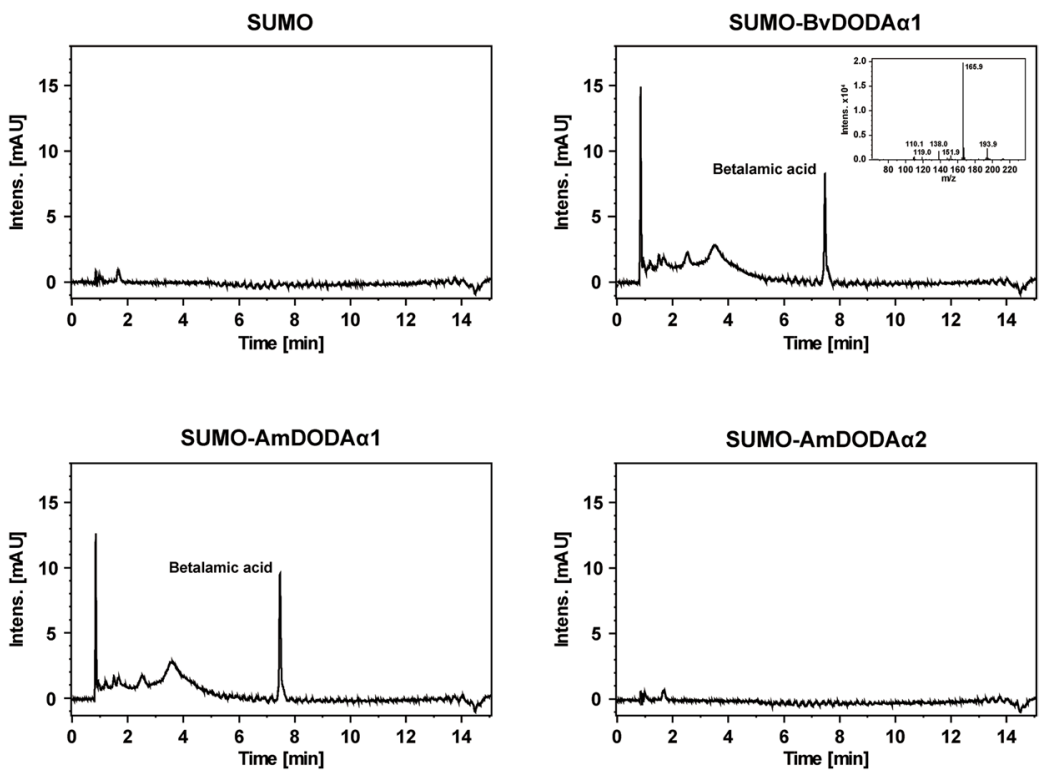

Figure 5. Examination of the L-DOPA 4,5-dioxygenase activity of $A m D O D A \alpha 1$ and $A m D O D A \alpha 2$ in vitro. (a) Crude extracts prepared from E. coli expressing SUMO-fused BvDODAa1, AmDODAa1, or AmDODAa2 were examined by Coomassie brilliant blue staining. Recombinant proteins were expressed in similar amounts. (b) Enzymatic reactions were conducted following the method described by Sasaki et $\mathrm{al}^{32}$ with some modifications. The reaction mixtures contained L-DOPA, ascorbic acid, and the crude extract prepared from $E$. coli harboring the $B v D O D A \alpha 1, A m D O D A \alpha 1$, or $A m D O D A \alpha 2$ gene. Yellow color derived from betalamic acid was examined to evaluate the enzymatic activity of L-DOPA 4,5-dioxygenase. (c) Elution profiles of the in vitro reactions were performed by LC-MS/MS. Betalamic acid was confirmed by a mass fragmentation profile of the peak at a retention time of $7.5 \mathrm{~min}$.

biosynthesis in Caryophyllales ${ }^{16,20,30,36,37}$. However, numerous duplication events have led to difficulty in elucidating the functional activities of key enzymes in betalain-pigmented species through annotation ${ }^{10}$. For example, duplication events gave rise to two major clades of DODA homologues, DODA $\alpha$ and DODA $\beta$, but only one gene paralog in the DODAa clade of each species exhibits high levels of L-DOPA 4,5-dioxygenase activity ${ }^{12,21}$. Thus, it is necessary to examine the possible involvement of annotated genes in betalain biosynthesis on the basis of experimental evidence. In this study, the AmDODA $\alpha 1$ and $A m D O D A \alpha 2$ genes, which belong to the DODA $\alpha$ clade according to phylogenetic construction and LOGO analysis (Fig. 4b,c), were identified in A. tricolor through transcriptome analysis (Supplementary Fig. S4, Table S4). Based on the heterologous engineering of betalain pigments in N. benthamiana and in vitro biochemical studies (Figs. 3b, 5b), we report that AmDODAal displayed a high level of L-DOPA 4,5-dioxygenase activity to produce betalamic acid, but such activity was barely detectable for AmDODAa2. These results indicate that at least one duplication event has occurred in the DODAa lineage of $A$. tricolor, and the primary function of AmDODAa2 remains to be further studied.

Betalains are composed of betacyanins and betaxanthins. In contrast to betaxanthins, which are derived from betalamic acid via spontaneous condensation with amino acids or other amines, a large number of betacyanins are composed of betanidin conjugated with glycosyl moieties ${ }^{9,10}$. We characterized the function of AmcDOP$A 5 G T$, a cyclo-DOPA 5-O-glucosyltransferase gene, through the heterologous engineering of betalain pigments in N. benthamiana. The coexpression of AmCYP76AD1, AmDODA $\alpha 1$, and AmcDOPA5GT enabled the production of high levels of betalain pigments with a dark red color (Fig. 6a). In contrast, low production of betalain pigments was observed when $A m C Y P 76 A D 1$ and $A m D O D A \alpha 1$ were coexpressed (Fig. 6a). Our results suggest the importance of AmcDOPA5GT in the glycosylation reaction during betalain biosynthesis in A. tricolor. In fact, the metabolic pathway of betalain biosynthesis is very complex due to multiple glycosylation steps, and different betacyanins have been identified ${ }^{10,38}$. For example, betanin, the most common betacyanin, is not only produced by cyclo-DOPA 5-O-glucosyltransferase but is also produced by betanidin 5-O-glucosyl-transferase through the glycosylation of betanidin ${ }^{39,40}$. In this study, AmB5GT, a betanidin 5-O-glucosyl-transferase gene, was also identified through comparative transcriptome analyses (Supplementary Table S4). Although AmcDOPA5GT showed higher expression levels than AmB5GT in both AMR and AMG (Supplementary Table S4), it remains to be determined which of the two glycosylation routes is more important for the formation of betanin in A. tricolor.

Recently, betalain biosynthesis in different pitaya species, such as Hylocereus polyrhizus, Hylocereus costaricensis, Hylocereus undatus, and Hylocereus megalanthus, has been intensively studied through comparative transcriptome analysis ${ }^{36,37,41,42}$. However, further studies remain to be conducted to provide experimental evidence and strengthen the understanding of the roles of candidate genes in betalain biosynthesis. Here, complementation assays conducted through the heterologous engineering of betalain pigments in nonbetalain-producing plants 
a

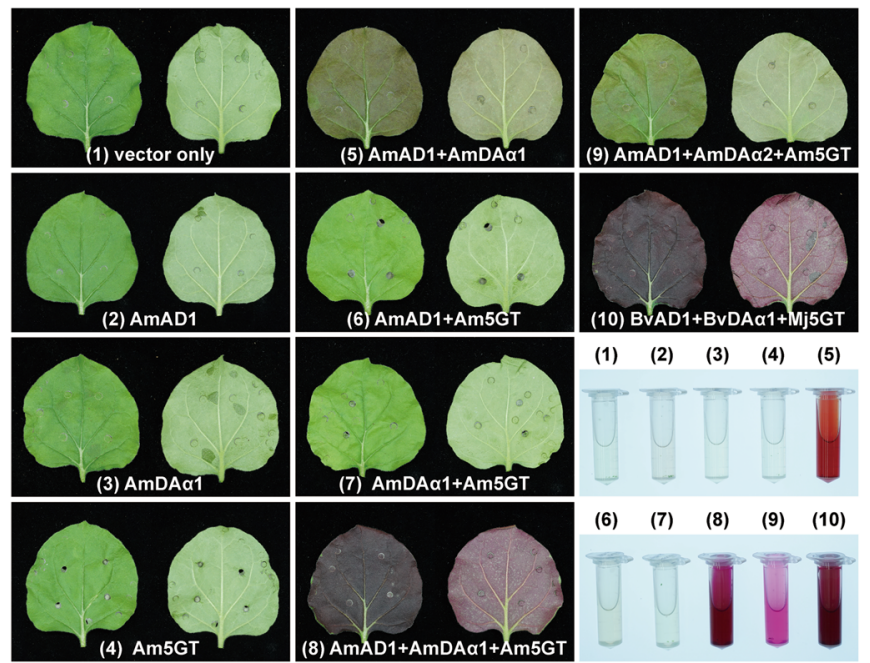

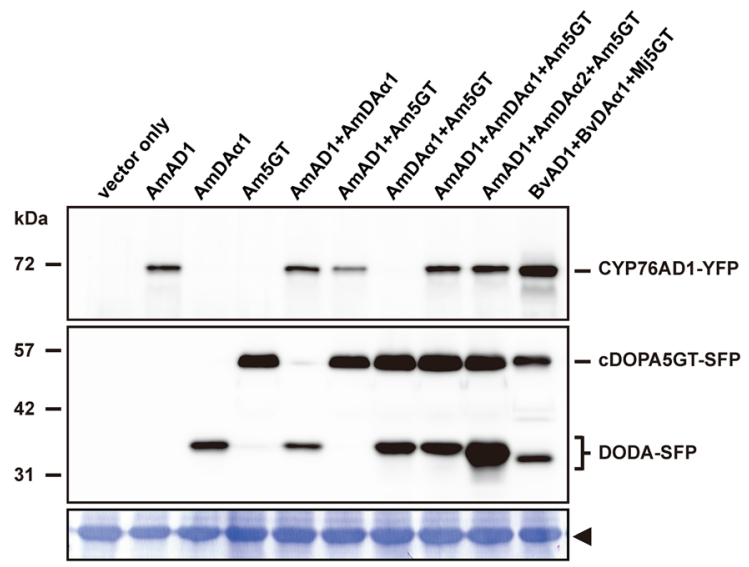

b
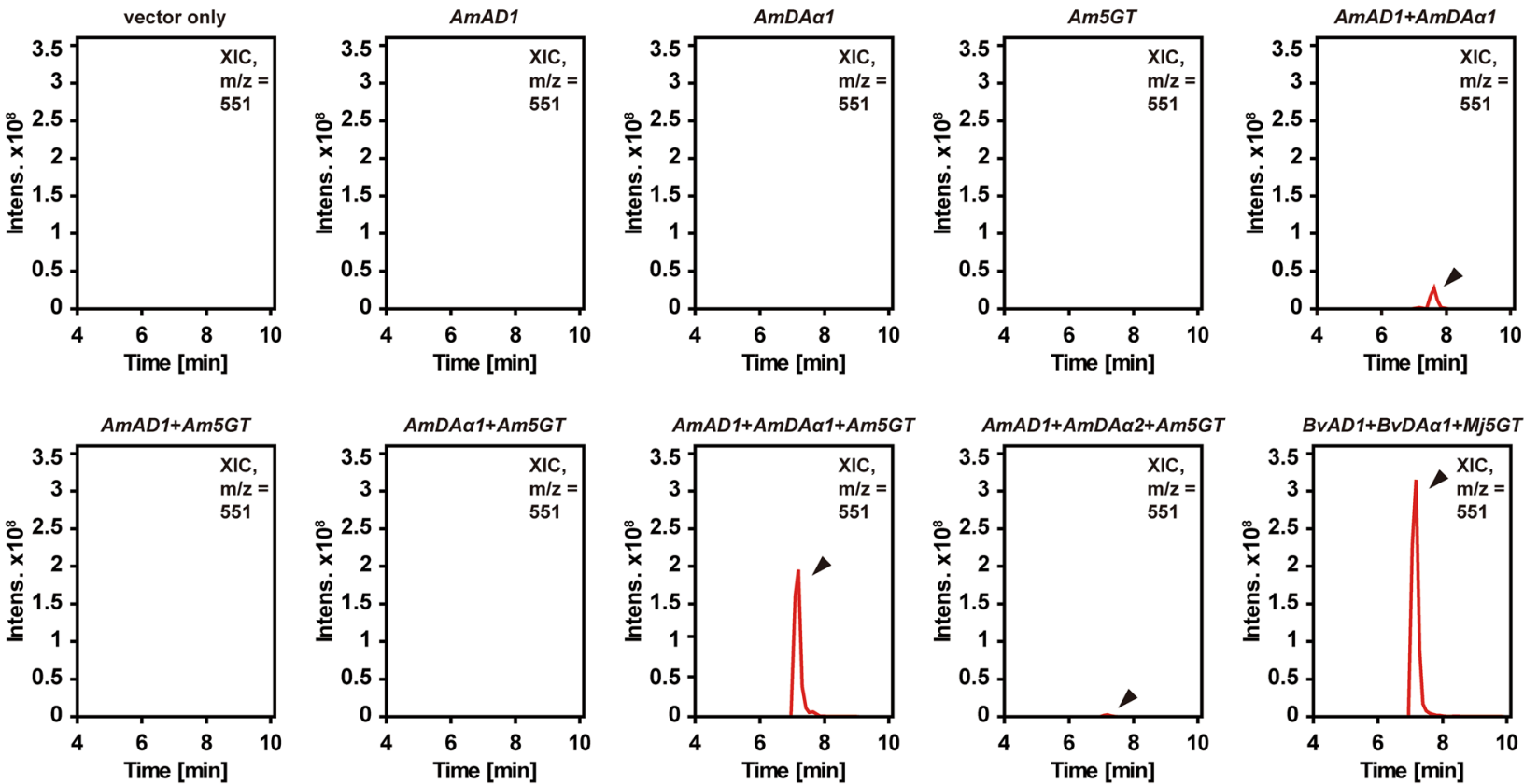

Figure 6. Reconstruction of the core betalain biosynthesis pathway of A. tricolor in N. benthamiana by agroinfiltration for the heterologous engineering of betalain pigments. (a) N. benthamiana leaves coinfiltrated with Agrobacterium harboring plasmids for the expression of BvCYP76AD1-YFP (BvAD1), BvDODA 1 1-SFP

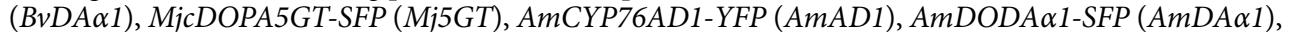
$A m D O D A \alpha 2-S F P$ (AmDA 2 ), and AmcDOPA5GT-SFP (Am5GT). Both the adaxial (left) and abaxial (right) sides of leaves are presented in each panel. Bottom right corner indicated the betalain pigments produced in $N$. benthamiana. (b) Extracted betalain pigments were examined for betanin content by LC-MS/MS analysis. Shown are XICs of masses corresponding to betanin $(\mathrm{m} / \mathrm{z}=551)$. Time, retention time (min). (c) Western blotting assays were conducted to examine the expression levels of YFP-tagged CYP76AD1 (upper panel), SFPtagged DODA (middle panel), and SFP-tagged cDOPA5GT (middle panel) using antibodies against the YFP- or FLAG (SFP)-tag. As a loading control, the large subunit of Rubisco visualized with Coomassie brilliant blue staining is indicated by the arrowhead (lower panel).

provided a solution for the easy and rapid comparison of the functional activities of genes involved in the core betalain biosynthesis pathway between betalain-pigmented species of Caryophyllales. Using the coexpression of $B v C Y P 76 A D 1, B v D O D A \alpha 1$, and MjcDOPA5GT in $N$. benthamiana as a positive control, the functional activities of $A$. tricolor genes responsible for betalain synthesis could be compared through a series of complementation assays (Fig. 3b-d). We showed that comparable amounts of betalain pigments were observed when the functional activities of positive genes were individually replaced with AmCYP76AD1, AmDODA $\alpha 1$, and AmcDOP$A 5 G T$ in transient coexpression assays (Fig. $3 \mathrm{~b}-\mathrm{d}$ ). Our results indicate that $A m C Y P 76 A D 1, A m D O D A \alpha 1$, and 
AmcDOPA5GT exhibit high tyrosinase, L-DOPA 4,5-dioxygenase, and cyclo-DOPA 5-O-glucosyltransferase activities, respectively, which are similar to those in B. vulgaris and M. jalapa. Accordingly, in vitro biochemical studies demonstrated that AmDODAa1 displayed comparable L-DOPA 4,5-dioxygenase activity to BvDODAa1 in producing betalamic acid (Fig. 5b). These results provide novel insights into betalain biosynthesis and evolution in A. tricolor.

In conclusion, a comparative transcriptome analysis combined with functional and enzymatic studies were performed to reveal the core betalain biosynthesis pathway of $A$. tricolor. The heterologous engineering of betalain pigments through the coexpression of AmCYP76AD1, AmDODA $\alpha 1$, and AmcDOPA5GT in N. benthamiana enabled the production of high amounts of betalain pigments with a red-violet color similar to those in the red-leaf cultivar of $A$. tricolor. Although the metabolic pathway of betalain biosynthesis is very complex, the core betalain biosynthesis pathway of $A$. tricolor constructed here not only provides a basal framework for examining genes related to betalain biosynthesis within the species of Amaranthaceae but also sheds light on the evolution of the betalain biosynthesis pathway in Caryophyllales.

\section{Methods}

Plant materials and growth conditions. A. tricolor, B. vulgaris, M. jalapa, and N. benthamiana plants were grown at $26^{\circ} \mathrm{C}$ in a semicontrolled walk-in chamber under a 16:8-h light:dark photoperiod. Soil (Jiffy) mixed with vermiculite and pearlstone was used. Seeds of $A$. tricolor cv. Hung Hsien (red-leaf cultivar) and $A$. tricolor cv. Pai Hsien (green-leaf cultivar) were purchased from KNOWN-YOU SEED CO., LTD.

Betalain pigment extraction and measurement. For betalain pigment measurement, betalain contents were determined as described previously with some modification ${ }^{43}$. Briefly, leaves of seedlings were collected and ground into powder in liquid nitrogen. Betalain pigments were extracted with extraction solution (methanol:chloroform: $\mathrm{H}_{2} \mathrm{O}$ [1:2:1]). After centrifugation, the upper (hydrophilic) layer was collected to measure the absorbance at $538 \mathrm{~nm}$ and $476 \mathrm{~nm}$ for betacyanins and betaxanthins, respectively. The relative betalain content was calculated with the following equation: $\left.\left(A_{538}+A_{476}\right) / \mathrm{gram}\right)$.

Plasmid construction. All plasmid constructs were generated using standard restriction site reconstruc-

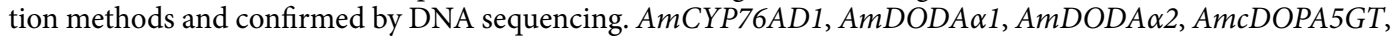
$B v C Y P 76 A D 1, B v D O D A \alpha 1$, and MjcDOPA5GT were amplified from A. tricolor, B. vulgaris, or M. jalapa cDNA libraries using AccuPrime pfx DNA polymerase (Invitrogen). For the transient expression of C-terminal YFPor FLAG (SFP)-tagged proteins in N. benthamiana, PCR products encoding AmCYP76AD1, AmDODA $\alpha$, $A m D O D A \alpha 2$, AmcDOPA5GT, BvCYP76AD1, BvDODA $\alpha 1$, and MjcDOPA5GT were subcloned into pBA-C-SFP or pBA-C-YFP vectors under the control of a Cauliflower mosaic virus (CaMV) $35 \mathrm{~S}$ promoter ${ }^{44}$. To produce $\mathrm{N}$-terminal SUMO-tagged recombinant proteins, PCR products encoding AmDODA $1, A m D O D A \alpha 2$, and $B v D O D A \alpha 1$ were subcloned into the pET-SUMO (Invitrogen) vector ${ }^{45}$. For the VIGS assay, a cDNA fragment of $A m C Y P 76 A D 1$ was amplified and subcloned into the pTRV2 vector ${ }^{46}$. The primer sequences used for plasmid construction are listed in Supplementary Table S5.

Quantitative real-time polymerase chain reaction (qRT-PCR) and statistical analysis. TRIzol ${ }^{\mathrm{m}}$ (Invitrogen)-extracted total RNA was reverse transcribed using SuperScript III First-Strand Synthesis SuperMix (Invitrogen) according to the manufacturer's instructions. Briefly, each sample was prepared from the leaves of three biologically distinct 3-week-old or 4-week-old $A$. tricolor plants. Then, cDNA was synthesized from $1 \mu \mathrm{g}$ of total RNA using a mixture of random hexamers and oligo $(\mathrm{dT})_{20}$ under the following conditions: $25^{\circ} \mathrm{C}$ for $10 \mathrm{~min}$, followed by $50^{\circ} \mathrm{C}$ for $40 \mathrm{~min}$. The cDNA was employed as a template for qRT-PCR using the KAPA SYBR Fast qPCR Kit (Kapa Biosystems). Three technical replicates were performed on a CFX96 $6^{\text {ma }}$ Real-time System (Bio-Rad) under the following conditions: $95^{\circ} \mathrm{C}$ for $3 \mathrm{~min}$, followed by 40 cycles of $95{ }^{\circ} \mathrm{C}$ for $10 \mathrm{~s}$ and $55^{\circ} \mathrm{C}$ for $30 \mathrm{~s}$. The expression levels of selected genes were determined by normalization to the reference gene Actin. Statistically significant differences were determined using Student's $t$-test in SPSS version 20.0. The primer sequences employed for qRT-PCR analyses are listed in Supplementary Table S1. PCR analyses using genomic DNA extracted from AMR and AMG as a template were performed to confirm the specificity of the primers (Supplementary Fig. S7).

Transient coexpression assay and western blotting. Plasmids for the transient expression of AmCYP76AD1-YFP, AmDODAa1-SFP, AmDODAa2-SFP, AmcDOPA5GT-SFP, BvCYP76AD1-YFP, BvDODAa1SFP, or MjcDOPA5GT-SFP were transformed into the Agrobacterium tumefaciens strain ABI. C-terminal tagged proteins were coexpressed using a mixture of A. tumefaciens carrying the desired constructs in N. benthamiana leaves by agroinfiltration following the method described previously ${ }^{47}$. After three days, the infiltrated leaves were photographed and ground into a powder in liquid nitrogen for total cell extract preparation. Briefly, $0.1 \mathrm{~g}$ of sample powder was added to $0.2 \mathrm{ml}$ of $2.5 \times \mathrm{SDS}$ sample buffer ( $5 \mathrm{mM}$ EDTA, $5 \%$ SDS, $0.3 \mathrm{M}$ Tris-HCl, $\mathrm{pH} 6.8$, $20 \%$ glycerol, $1 \% \beta$-mercaptoethanol, and bromophenol blue), which was then heated at $95{ }^{\circ} \mathrm{C}$ in a dry bath for $10 \mathrm{~min}$. After centrifugation at $13,000 \times g$ for $10 \mathrm{~min}$, the supernatant was obtained, and total proteins were separated by SDS-PAGE. Western blotting assays were performed to monitor protein levels using specific polyclonal and monoclonal antibodies against YFP- and FLAG-tag, respectively. Chemiluminescence signals generated by ECL reagents (PerkinElmer) were captured with an ImageQuant LAS 4000 mini imager (GE Healthcare). All experiments were repeated at least three times using biologically distinct samples prepared from two infiltrated leaves. 
In vitro L-DOPA 4,5-dioxygenase activity assay and liquid chromatography-tandem mass spectrometry (LC-MS/MS) analysis. An in vitro L-DOPA 4,5-dioxygenase activity assay was performed according to the method described previously with some modification ${ }^{32}$. Briefly, plasmids for the expression of N-terminal SUMO-tagged $A m D O D A \alpha 1, A m D O D A \alpha 2$ and $B v D O D A \alpha 1$ were transformed into Escherichia coli strain BL21 (DE3). The transformants were grown in $50 \mathrm{ml} \mathrm{LB}$ medium, and the recombinant proteins were induced with $0.2 \mathrm{mM} \mathrm{IPTG}$ at $22^{\circ} \mathrm{C}$ for $16 \mathrm{~h}$. Harvested cells were washed, resuspended, and disrupted by sonication in $50 \mathrm{mM}$ sodium phosphate buffer ( $\mathrm{pH}$ 7.0). The crude extract (supernatant) was used for the enzyme activity assay after centrifugation at $14,000 \times g$ for $15 \mathrm{~min}$. The amount of recombinant protein was quantified with Protein Assay Reagent (Bio-Rad) and via Coomassie blue staining SDS-PAGE with BSA as the standard. Basically, the reaction $(100 \mu \mathrm{l})$ was performed with the crude extract containing $8 \mu \mathrm{g}$ DODA protein, $27 \mathrm{mM}$ ascorbic acid, and $6.75 \mathrm{mM} \mathrm{L}-\mathrm{DOPA}$ at $30^{\circ} \mathrm{C}$ for $5 \mathrm{~min}$.

LC-MS/MS was performed using a Dionex UltiMate 3000 system (Thermo Fisher Scientific) linked with an amaZon speed-ion trap mass spectrometer (Bruker). Betalamic acid was detected on a Waters BEH shield RP18 column with two eluting solvent systems: (A) $\mathrm{H}_{2} \mathrm{O}$ with $0.1 \%$ formic acid, (B) $100 \%$ acetonitrile. The gradient elution program was set as follows: $0-3 \mathrm{~min}(100 \% \mathrm{~A}), 9 \mathrm{~min}(55 \% \mathrm{~A}$ and $45 \% \mathrm{~B}), 12-13 \mathrm{~min}(100 \% \mathrm{~B})$. The flow rate was $0.3 \mathrm{ml} \mathrm{min}^{-1}$, and the detector wavelength was $424 \mathrm{~nm}$. The electrospray ionization mass parameters were set as follows: $4.5 \mathrm{kV}$ capillary, $500 \mathrm{~V}$ end plate offset voltage, 40.0 psi nebulizer pressure, $8.01 \mathrm{~min}^{-1} \mathrm{dry}$ gas, and $230^{\circ} \mathrm{C}$ dry temperature. The measurement was operated in multiple reaction-monitoring (MRM) with the positive ion mode. The MRM was set $182 \rightarrow 165 \mathrm{~m} / \mathrm{z}$ to detect tyrosine, $198 \rightarrow 181 \mathrm{~m} / \mathrm{z}$ to detect $L$-DOPA, $212 \rightarrow 166 \mathrm{~m} / \mathrm{z}$ to detect betalamic acid, $389 \rightarrow 345 \mathrm{~m} / \mathrm{z}$ to detect betanidin, and $551 \rightarrow 389 \mathrm{~m} / \mathrm{z}$ to detect betanin.

Next-generation sequencing and MA plot. To perform next-generation sequencing, aerial tissues derived from three biologically distinct 3-week-old A. tricolor plants were collected. Total RNA was extracted using the RNeasy Plant Mini Kit (Qiagen) according to the manufacturer's instructions. RNA quality was examined via $1.2 \%$ (wt/vol) formaldehyde gel electrophoresis and with an Experion RNA analysis kit (Bio-Rad, Munich). Only high-quality RNA was used for next-generation sequencing performed on the Illumina HiSeq 4000 platform with 150 paired-end reads. For each dataset (AMR and AMG), 100 million reads were generated, and de novo assembly was performed with the Trinity tool. The assembled transcripts were annotated with BlastX in UniProt. Gene expression levels were normalized as FPKM values, and differentially expressed genes were identified according to an FDR $<0.05$ and $\operatorname{logFC}>2$ or $<-2$ (Supplementary Tables S2, S3). An MA plot was generated based on the average concentration $(\log \mathrm{CPM})$ and fold-change $(\log \mathrm{FC})$ values to show the relative abundances of transcripts between AMR and AMG.

Phylogenetic tree reconstruction and LOGO analysis. Phylogenetic trees were reconstructed using MEGA-X software based on the protein sequence comparisons of CYP76AD and DODA homologues from different betalain-producing species. Multiple sequence alignments were performed using the MUSCLE program and were processed to generate a maximum likelihood phylogenetic tree via the Jones-Taylor-Thornton (JTT) model with bootstrapping to perform molecular evolutionary analysis. The numbers at the branch points are bootstrap values representing the percentages of replicate trees based on 1000 repeats. LOGO analyses were performed via WebLogo (http://weblogo.berkeley.edu/logo.cgi) based on selected conserved amino acids of CYP76AD and DODA homologues reported previously,12,21,48. The species, families, and accession numbers of CYP76AD and DODAa homologues are available in Supplementary Table S6.

\section{Data Availability}

Sequencing data generated for this study are deposited at Short Read Archive with the accession code SRR15044103.

Received: 18 November 2020; Accepted: 2 March 2021

Published online: 17 March 2021

\section{References}

1. Polturak, G. \& Aharoni, A. "La Vie En Rose”: Biosynthesis, sources, and applications of betalain pigments. Mol. Plant 11, 7-22 (2018).

2. Jain, G. \& Gould, K. S. Are betalain pigments the functional homologues of anthocyanins in plants?. Environ. Exp. Bot. 119, 48-53 (2015).

3. Li, G., Meng, X., Zhu, M. \& Li, Z. Research progress of betalain in response to adverse stresses and evolutionary relationship compared with anthocyanin. Molecules 24, 3078. https://doi.org/10.3390/molecules24173078 (2019).

4. Marchesini, V. A., Yin, C., Colmer, T. D. \& Veneklaas, E. J. Drought tolerances of three stem-succulent halophyte species of an inland semiarid salt lake system. Funct. Plant Biol. 41, 1230-1238 (2014).

5. Park, S. C. et al. Overexpression of the IbMYB1 gene in an orange-fleshed sweet potato cultivar produces a dual-pigmented transgenic sweet potato with improved antioxidant activity. Physiol. Plant 153, 525-537 (2015).

6. Polturak, G. et al. Engineered gray mold resistance, antioxidant capacity, and pigmentation in betalain-producing crops and ornamentals. Proc. Natl. Acad. Sci. U.S.A. 114, 9062-9067 (2017).

7. Gengatharan, A., Dykes, G. A. \& Choo, W. S. Betalains: natural plant pigments with potential application in functional foods. LWT - Food Sci. Technol. 64, 645-649 (2015).

8. Stintzing, F. C. \& Carle, R. Functional properties of anthocyanins and betalains in plants, food, and in human nutrition. Trends Food Sci. Technol. 15, 19-38 (2004).

9. Brockington, S. F. et al. Lineage-specific gene radiations underlie the evolution of novel betalain pigmentation in Caryophyllales. New Phytol. 207, 1170-1180 (2015). 
10. Timoneda, A. et al. The evolution of betalain biosynthesis in Caryophyllales. New Phytol. 224, 71-85 (2019).

11. Lopez-Nieves, S. et al. Relaxation of tyrosine pathway regulation underlies the evolution of betalain pigmentation in Caryophyllales. New Phytol. 217, 896-908 (2018).

12. Sheehan, H. et al. Evolution of 1-DOPA 4,5-dioxygenase activity allows for recurrent specialisation to betalain pigmentation in Caryophyllales. New Phytol. 227, 914-929 (2020).

13. Gandía-Herrero, F. \& García-Carmona, F. Biosynthesis of betalains: Yellow and violet plant pigments. Trends Plant Sci. 18, 334-343 (2013).

14. Takos, A. M. et al. Light-induced expression of a MYB gene regulates anthocyanin biosynthesis in red apples. Plant Physiol. 142, 1216-1232 (2006).

15. DeLoache, W. C. et al. An enzyme-coupled biosensor enables (S)-reticuline production in yeast from glucose. Nat. Chem. Biol. 11, 465-471 (2015).

16. Polturak, G. et al. Elucidation of the first committed step in betalain biosynthesis enables the heterologous engineering of betalain pigments in plants. New Phytol. 210, 269-283 (2016).

17. Christinet, L., Burdet, F. X., Zaiko, M., Hinz, U. \& Zrÿd, J.-P. Characterization and functional identification of a novel plant 4, 5-extradiol dioxygenase involved in betalain pigment biosynthesis in Portulaca grandiflora. Plant Physiol. 134, 265-274 (2004).

18. Hatlestad, G. J. et al. The beet R locus encodes a new cytochrome P450 required for red betalain production. Nat. Genet. 44, 816-820 (2012).

19. Sasaki, N., Adachi, T., Koda, T. \& Ozeki, Y. Detection of UDP-glucose: cyclo-DOPA 5-O-glucosyltransferase activity in four o'clocks (Mirabilis jalapa L.). FEBS Lett. 568, 159-162 (2004).

20. Polturak, G. et al. Transcriptome and metabolic profiling provides insights into betalain biosynthesis and evolution in Mirabilis jalapa. Mol. Plant 11, 189-204 (2018).

21. Bean, A. et al. Gain-of-function mutations in beet DODA 2 identify key residues for betalain pigment evolution. New Phytol. 219, 287-296 (2018).

22. Guerrero-Rubio, M. A., Escribano, J., García-Carmona, F. \& Gandía-Herrero, F. Light emission in betalains: from fluorescent flowers to biotechnological applications. Trends Plant Sci. 25, 159-175 (2020).

23. Polturak, G. \& Aharoni, A. Advances and future directions in betalain metabolic engineering. New Phytol. 224, 1472-1478 (2019).

24. Cabanes, J., Gandía-Herrero, F., Escribano, J., García-Carmona, F. \& Jiménez-Atiénzar, M. Fluorescent bioinspired protein labeling with betalamic acid. Derivatization and characterization of novel protein-betaxanthins. Dyes Pigm. 133, 458-466 (2016).

25. Tian, Y.-S. et al. Metabolic engineering of rice endosperm for betanin biosynthesis. New Phytol. 225, 1915-1922 (2020).

26. Miguel, M. G. Betalains in some species of the Amaranthaceae family: a review. Antioxidants 7, 53. https://doi.org/10.3390/antio x7040053 (2018)

27. Sarker, U. \& Oba, S. Antioxidant constituents of three selected red and green color Amaranthus leafy vegetable. Sci. Rep. 9, 1-11 (2019).

28. Khandaker, L. \& Oba, S. Influence of cultivar and growth stage on pigments and processing factors on betacyanins in red Amaranth (Amaranthus tricolor L.). Food Sci. Technol. Int. 15, 259-265 (2009).

29. Adhikary, D., Khatri-Chhetri, U., Tymm, F. J., Murch, S. J. \& Deyholos, M. K. A virus-induced gene-silencing system for functional genetics in a betalainic species, Amaranthus tricolor (Amaranthaceae). Appl. Plant Sci. 7, e01221. https://doi.org/10.1002/aps3. 1221 (2019).

30. Liu, S. et al. RNA-sequencing analysis reveals betalains metabolism in the leaf of Amaranthus tricolor L. PLoS ONE 14, e0216001. https://doi.org/10.1371/journal.pone.0216001 (2019).

31. Zheng, X. et al. Cloning and expression analysis of betalain biosynthesis genes in Amaranthus tricolor. Biotechnol. Lett. 38, 723-729 (2016).

32. Sasaki, N. et al. Detection of DOPA 4,5-dioxygenase (DOD) activity using recombinant protein prepared from Escherichia coli cells harboring cDNA encoding DOD from Mirabilis jalapa. Plant Cell Physiol. 50, 1012-1016 (2009).

33. Hwang, H.-H. et al. Characterization and host range of five tumorigenic Agrobacterium tumefaciens strains and possible application in plant transient transformation assays. Plant Pathol. 62, 1384-1397 (2013).

34. Strack, D. \& Schliemann, W. Bifunctional polyphenol oxidases: novel functions in plant pigment biosynthesis. Angew. Chem. Int. Ed. 40, 3791-3794 (2001).

35. Teng, X.-L., Chen, N. \& Xiao, X.-G. Identification of a catalase-phenol oxidase in betalain biosynthesis in red amaranth (Amaranthus cruentus). Front. Plant Sci. 6, 1228. https://doi.org/10.3389/fpls.2015.01228 (2016).

36. Qingzhu, H. et al. Transcriptomic analysis reveals key genes related to betalain biosynthesis in pulp coloration of Hylocereus polyrhizus. Front. Plant Sci. 6, 1179. https://doi.org/10.3389/fpls.2015.01179 (2016).

37. Wu, Y. et al. Comparative transcriptome analysis combining SMRT-and Illumina-based RNA-Seq identifies potential candidate genes involved in betalain biosynthesis in pitaya fruit. Int. J. Mol. Sci. 21, 3288. https://doi.org/10.3390/ijms21093288 (2020).

38. Wybraniec, S., Jerz, G., Gebers, N. \& Winterhalter, P. Ion-pair high-speed countercurrent chromatography in fractionation of a high-molecular weight variation of acyl-oligosaccharide linked betacyanins from purple bracts of Bougainvillea glabra. J. Chromatogr. B Anal. Technol. Biomed. Life Sci. 878, 538-550 (2010).

39. Strack, D., Vogt, T. \& Schliemann, W. Recent advances in betalain research. Phytochemistry 62, 247-269 (2003).

40. Vogt, T. Substrate specificity and sequence analysis define a polyphyletic origin of betanidin 5- and 6-O-glucosyltransferase from Dorotheanthus bellidiformis. Planta 214, 492-495 (2002).

41. Xi, X. et al. Transcriptome analysis clarified genes involved in betalain biosynthesis in the fruit of red pitayas (Hylocereus costaricensis). Molecules 24, 445. https://doi.org/10.3390/molecules24030445 (2019).

42. Xie, F. et al. Transcriptomics-based identification and characterization of glucosyltransferases involved in betalain biosynthesis in Hylocereus megalanthus. Plant Physiol. Biochem. 152, 112-124 (2020).

43. von Elbe, J.H. Betalains. Curr. Protoc. Food Anal. Chem. 00: F3.1.1-F3.1.7 (2001).

44. Chang, S. H. et al. Alterations of plant architecture and phase transition by the phytoplasma virulence factor SAP11. J. Exp. Bot. 69, 5389-5401 (2018).

45. Tan, C. M. et al. Phytoplasma SAP11 alters 3-isobutyl-2-methoxypyrazine biosynthesis in Nicotiana benthamiana by suppressing NbOMT1. J. Exp. Bot. 67, 4415-4425 (2016).

46. Dinesh-Kumar, S. P., Anandalakshmi, R., Marathe, R., Schiff, M. \& Liu, Y. Virus-induced gene silencing. Methods Mol. Biol. 236, 287-294 (2003).

47. Leuzinger, K. et al. Efficient agroinfiltration of plants for high-level transient expression of recombinant proteins. J. Vis. Exp. 77, e50521. https://doi.org/10.3791/50521 (2013).

48. Sunnadeniya, R. et al. Tyrosine hydroxylation in betalain pigment biosynthesis is performed by cytochrome $\mathrm{P} 450$ enzymes in beets (Beta vulgaris). PLoS ONE 11, e0149417. https://doi.org/10.1371/journal.pone.0149417 (2016).

\section{Acknowledgements}

This work was financially supported (in part) by grants-in-aid from the Ministry of Science and Technology (MOST-109-2628-B-005-006) and the Advanced Plant Biotechnology Center from the Featured Areas Research 
Center Program within the framework of the Higher Education Sprout Project by the Ministry of Education (MOE) in Taiwan.

\title{
Author contributions
}

Y.-C.C. carried out most of the experiments; Y.-C.C. and Y.-L.C. designed and carried out the enzymatic assays. N.-W.T. performed the LC-MS analysis. C.-M.T., Y.-H.C., and P.-C.L. performed RNA-seq and plasmid constructions. Y.-C.L., L.-C.H. and S.-Y.W. contributed to the interpretation of data. J.-Y.Y. conceived and wrote the manuscript.

\section{Competing interests}

The authors declare no competing interests.

\section{Additional information}

Supplementary Information The online version contains supplementary material available at https://doi.org/ 10.1038/s41598-021-85486-x.

Correspondence and requests for materials should be addressed to J.-Y.Y.

Reprints and permissions information is available at www.nature.com/reprints.

Publisher's note Springer Nature remains neutral with regard to jurisdictional claims in published maps and institutional affiliations.

\begin{abstract}
Open Access This article is licensed under a Creative Commons Attribution 4.0 International License, which permits use, sharing, adaptation, distribution and reproduction in any medium or
format, as long as you give appropriate credit to the original author(s) and the source, provide a link to the Creative Commons licence, and indicate if changes were made. The images or other third party material in this article are included in the article's Creative Commons licence, unless indicated otherwise in a credit line to the material. If material is not included in the article's Creative Commons licence and your intended use is not permitted by statutory regulation or exceeds the permitted use, you will need to obtain permission directly from the copyright holder. To view a copy of this licence, visit http://creativecommons.org/licenses/by/4.0/.
\end{abstract}

(C) The Author(s) 2021, corrected publication 2021 